\title{
Withaferin A Inhibits Nuclear Factor- $\kappa$ B-Dependent Pro-Inflammatory and Stress Response Pathways in the Astrocytes
}

\author{
Francesca Martorana, Giulia Guidotti, Liliana Brambilla, and Daniela Rossi \\ Laboratory for Research on Neurodegenerative Disorders, IRCCS Fondazione Salvatore Maugeri, 27100 Pavia, Italy \\ Correspondence should be addressed to Daniela Rossi; daniela.rossi@fsm.it
}

Received 27 February 2015; Revised 1 July 2015; Accepted 2 July 2015

Academic Editor: Nicola Maggio

Copyright (C) 2015 Francesca Martorana et al. This is an open access article distributed under the Creative Commons Attribution License, which permits unrestricted use, distribution, and reproduction in any medium, provided the original work is properly cited.

\begin{abstract}
Several lines of evidence suggest that astrocytes play a key role in modulating the immune responses of the central nervous system (CNS) to infections, injuries, or pathologies. Yet, their contribution to these processes remains mostly elusive. Astroglia are endowed with a wide range of toll-like receptors (TLR) by which they can sense infectious agents as well as endogenous danger signals released by damaged cells. Here we demonstrate that the activation of astrocytic TLR4 by bacterial lipopolysaccharide (LPS) challenge can promote nuclear factor $\kappa \mathrm{B}(\mathrm{NF}-\kappa \mathrm{B})$-dependent induction of pro-inflammatory and stress response mediators, particularly Tumor Necrosis Factor $\alpha$ (TNF $\alpha$ ), cyclooxygenase 2 (COX-2), and inducible nitric oxide synthase (iNOS). Since the steroid lactone Withaferin A was described to inhibit NF- $\kappa$ B activity in different cell types, we next determined the impact of this natural compound towards the identified astrocytic signalling pathway. Innate immune activation was induced by stimulation of the LPS/TLR4 axis in spinal cord astrocytes. We provide evidence that both pre-treating and post-treating the cells with Withaferin A attenuate astrocytic NF- $\kappa$ B activity as well as the consequent production of TNF $\alpha$, COX-2, and iNOS induced by stimulation of the LPS/TLR4 pathway. This study suggests that Withaferin A may be an eligible candidate for the treatment of neuroinflammatory and stress conditions characterized by an important astrocytic input.
\end{abstract}

\section{Introduction}

Pathologies, infections, or injuries of the central nervous system (CNS) are often accompanied by an atypical inflammatory process that occurs locally in the brain parenchyma and does not involve the peripheral immune system, with only limited leukocyte infiltration. Such a localized response is defined "neuroinflammation" and primarily involves the cellular effectors of the CNS innate immune system [1,2]. While microglia are considered the archetypical resident innate immune cells in brain and spinal cord, there is growing evidence indicating that also astrocytes can play an active role in these processes [1-3]. Yet, their contribution has been so far much less investigated.

Astrocytes represent the major glial cell population in the CNS and constitute up to $50 \%$ of its volume. In physiological conditions, they perform various activities that are essential to maintain CNS homeostasis, being involved in synaptic transmission, brain energetics, and cerebrovascular regulation $[3,4]$. However, astrocytes can also sense microbial infections through the recognition of conserved motifs expressed by a wide range of pathogens. In addition, during injury or disease, they can react to endogenous "danger signals" that are typically released by damaged cells to alert the immune system [5]. The recognition of such molecules is enabled by the astrocytic expression of specific pattern-recognition receptors, including various members of the toll-like receptor (TLR) family $[1,2,6]$. Studies in vitro and in human postmortem tissues consistently reported low but detectable astrocytic expression of TLR4 [7-10], a receptor that confers cellular responsiveness to the endotoxin lipopolysaccharide (LPS), an integral component of the outer membranes of Gram-negative bacteria [11-13]. 
Stimulation of astrocytic TLR4 by LPS was described to trigger a signaling cascade that leads to the activation of nuclear factor $\kappa \mathrm{B}(\mathrm{NF}-\kappa \mathrm{B})$ [14], a multifunctional transcription factor that controls the expression of several proinflammatory and stress response mediators [15]. Such events may be relevant in the context of chronic neurological disorders, where sustained inflammatory reactions were consistently reported to contribute to neurodegeneration and disease progression [6]. Based on this, it is reasonable to postulate that the identification of novel NF- $\kappa \mathrm{B}$ inhibitors that limit these effects in the astrocytes is highly desirable and may be beneficial to prevent neurodegenerative processes and to ameliorate disease outcomes.

A natural compound that was consistently reported to inhibit NF- $\kappa$ B signaling in non-CNS cell types and pathologies is Withaferin $\mathrm{A}$, a steroidal lactone isolated from the roots of Withania somnifera [16-21]. Despite the fact that an anti-inflammatory activity of this molecule has been reported within the CNS in microglia [22-24], its impact on astroglial cells has been poorly investigated.

In the present study, we focused on astrocytes and we specifically addressed the effects of this natural compound in preventing the astrocytic activation of NF- $\kappa \mathrm{B}$, and the consequent production of potentially neurotoxic pro-inflammatory and stress response mediators.

\section{Methods}

2.1. Cell Cultures. The P0-17D cell line was previously generated by immortalization of neonatal mouse cortical astrocytes with the SV40 large T antigen [25]. Morphologically and biochemically, these cells resemble mature primary astrocytes [25]. P0-17D cells were routinely maintained in Dulbecco's modified Eagle's medium (DMEM, Life Technologies) supplemented with $10 \%$ fetal bovine serum (FBS, SigmaAldrich), $2 \mathrm{mM}$ glutamine and antibiotics (Euroclone). Primary astrocyte cultures (99\% GFAP-positive) were prepared from the spinal cord of newborn C57BL6/SJL mice, as previously described $[26,27]$. Once the cultures reached the confluence, they were replated at the optimal density in 24well plates with or without glass coverslips and maintained in Minimal Essential Medium (MEM, Life Technologies) supplemented with $10 \%$ FBS.

2.2. Transfection and Luciferase Enzymatic Assay. P0-17D cells $\left(1 \times 10^{4}\right.$ cells/well $)$ or primary astrocytes $\left(2 \times 10^{4}\right.$ cells/well) were grown in 24-well plates and transiently co-transfected with the pGL4.32[luc2P/NF- $\kappa \mathrm{B}-\mathrm{RE} /$ Hygro] reporter vector (Promega) (hereafter referred to as "NF- $\kappa \mathrm{B}$ RE-Luc transgene"), which contains the firefly luciferase gene under the control of the NF- $\kappa$ B-responsive elements, and the normalization plasmid pGL4.74[hRluc/TK] (Promega) containing the Renilla luciferase gene (firefly: Renilla vector ratio, $50: 1$ ). Alternatively, cells were co-transfected with the pLucTKS3 STAT3 reporter vector, which contains signal transducer and activator of transcription 3 (STAT3)-driven firefly luciferase, the Renilla luciferase reporter vector and the pSG5-STAT3 $\alpha$ vector, a plasmid encoding the STAT3 $\alpha$ protein activator. Twenty-four hours after the transfection, cells were preincubated in the absence or in the presence of increasing concentrations of Withaferin A $(0.1,0.5$, $1 \mu \mathrm{M}$; Tocris) for 1 hour and, then, stimulated with LPS $(1 \mu \mathrm{g} / \mathrm{mL}$; Sigma-Aldrich) for another 6 hours. Cells were subsequently lysed by using a Passive Lysis Buffer (Promega) and luciferase enzymatic activity, considered as surrogate of $\mathrm{NF}-\kappa \mathrm{B}$ or STAT3 transcriptional activity, was determined. Firefly and Renilla luciferase signals were measured using a Dual-Luciferase Reporter Assay System (Promega) according to manufacturer's instructions, and the normalized firefly luminescence/Renilla luminescence ratio was calculated per each sample.

2.3. Pharmacological Treatments. Pre-treatment experiments were carried out by preincubating the cells for 1 hour in the absence or in the presence of SC-514 (10 $\mu \mathrm{M}$; Selleckchem) or Withaferin A $(0.5 \mu \mathrm{M})$, followed by a stimulation with LPS $(1 \mu \mathrm{g} / \mathrm{mL})$ for 6 hours. In post-treatment experiments, cells were exposed to LPS ( $1 \mu \mathrm{g} / \mathrm{mL} ; 6$ hours). Two hours after the start of the incubation, medium was supplemented with or without Withaferin $\mathrm{A}(0.5 \mu \mathrm{M})$ and incubation was continued for additional 4 hours. The expression levels of TNF $\alpha, \mathrm{COX}$ 2 , and iNOS mRNAs were then analyzed by quantitative reverse transcription-polymerase chain reaction (RT-qPCR) as described below.

2.4. Quantitative RT-PCR. The expression of TLR4, TNF $\alpha$, COX-2, and iNOS was determined by RT-qPCR in the P0$17 \mathrm{D}$ cell line, primary astrocytes, or spinal cord from adult C57BL6/SJL mice, as follows. Total RNA from cell or tissue lysates was extracted using RNeasy Mini Kit (QIAGEN) according to the manufacturer's guidelines. One microgram of total RNA was reverse-transcribed using iScript cDNA Synthesis Kit according to the manufacturer's instructions (BIO-RAD). Subsequent quantitative PCR was performed with the SsoFast EvaGreen Supermix on a CFX96 Real-Time PCR Detection System (BIO-RAD). Quantification of TLR4, TNF $\alpha$, COX-2, and iNOS mRNAs was normalized to the housekeeping gene hypoxanthine guanine phosphoribosyl transferase (HPRT) expression. The sequences of all RTqPCR primers are listed in Table 1.

2.5. Immunocytochemistry. After LPS treatment, astrocytes seeded on glass coverslips were fixed in $4 \%$ paraformaldehyde for 20 minutes and subsequently immunolabelled for NF$\kappa \mathrm{B}$ p65 using a rabbit polyclonal antibody (Abcam). Nuclei were stained with Hoechst 33342 (Sigma-Aldrich). Images were captured using a 40x objective lens on a DM5000 B microscope (Leica Microsystem) equipped with a digital camera DFC 310 FX (Leica Microsystem).

\section{Results and Discussion}

To set the experimental conditions for studying the activity on astrocytes of the steroid lactone Withaferin A, we initially established a cell-based assay by using the immortalized P017D astrocytic cell line [25]. The potential responsiveness to 
TABLE 1: Overview over primer sequences for RT-qPCR.

\begin{tabular}{lll}
\hline Gene & Forward primer & Reverse primer \\
\hline TLR4 & GGACTCTGATCATGGCACTG & CTGATCCATGCATTGGTAGGT \\
TNF $\alpha$ & AATGGCCTCCCTCTCATCAGTT & CCACTTGGTGGTTTGCTACGA \\
COX-2 & AAGCGAGGACCTGGGTTCA & AAGGCGCAGTTTATGTTGTCTGT \\
iNOS & GTTCTCAGCCCAACAATACAAGA & GTGGACGGGTCGATGTCAC \\
HPRT & TGAATCACGTTTGTGTCATTA & TTCAACTTGCGCTCATCTTAG \\
\hline
\end{tabular}

LPS/TLR4 signalling of P0-17D cortical astrocytes was firstly evaluated by determining the expression of TLR4 in these cells by RT-qPCR. Quantification of TLR4-encoding transcripts confirmed significantly higher levels of the receptorencoding mRNA in the P0-17D cells when compared to those in the mouse spinal cord (Figure 1). Since stimulation of TLR4 can trigger NF- $\kappa \mathrm{B}$ transcriptional activity in a vast array of cell types, we next decided to transiently transfect the P0-17D astrocytic cells with the reporter transgene NF- $\kappa \mathrm{B}-$ $\mathrm{RE}-L u c$, a minimal promoter-driven firefly luciferase reporter vector containing five copies of the NF- $\kappa \mathrm{B}$-responsive element. Luciferase enzymatic activity was determined, as surrogate of NF- $\kappa \mathrm{B}$ transcriptional activity, after treating the cells in the absence or in the presence of LPS $(1 \mu \mathrm{g} / \mathrm{mL})$ for 6 hours. We found that LPS-induced NF- $\kappa$ B transcriptional activity is significantly enhanced when compared with control conditions, that is, the corresponding culture type challenged with saline (Figure 2(a)). Conversely, the bacterial endotoxin was unable to trigger the transcriptional activity of the signal transducer and activator of transcription 3 (STAT3) in cells transfected with a STAT3-driven luciferase reporter vector (Figure 2(b)). The fact that LPS resulted incapable of activating STAT3 in the P0-17D astrocytic cells is at variance with previous studies describing the activation of astrocytic STAT3 in vitro and in vivo, upon stimulation with the bacterial lipopolysaccharide [28-32]. Yet, all these reports determined the activation of STAT3 by detecting either the phosphorylation or the nuclear translocation of the transcription factor. The indirect nature of these approaches makes difficult to draw definitive conclusions on the actual state of STAT3 activation and transcriptional activity. By contrast, taking advantage of the luciferase reporter assay mentioned above, we could reliably measure the transcriptional activity of STAT3 in a more direct, dynamic, and quantitative fashion. Based on these results, we can conclude that, in P0-17D astrocytes, the treatment with LPS preferentially activates the NF- $\kappa$ B transcriptional pathway, rather than STAT3 signalling.

In the next set of experiments, we exploited the P0-17D astrocyte-based luciferase reporter assay to screen the NF$\kappa \mathrm{B}$ inhibitory effects of Withaferin A. Cells were pre-treated with increasing concentrations of Withaferin $\mathrm{A}(0.1,0.5$, and $1 \mu \mathrm{M})$ for 1 hour and then incubated with LPS $(1 \mu \mathrm{g} / \mathrm{mL})$ for additional 6 hours. As shown in Figure 2(a), LPS-induced NF- $\kappa$ B-driven luciferase activity was significantly reduced by Withaferin $\mathrm{A}$ in a dose-dependent manner, reaching the maximum inhibitory effect at $0.5 \mu \mathrm{M}$ (Figure 2(a)). By contrast, Withaferin A alone did not significantly affect the basal activity of NF- $\kappa$ B (Figure 2(a)).

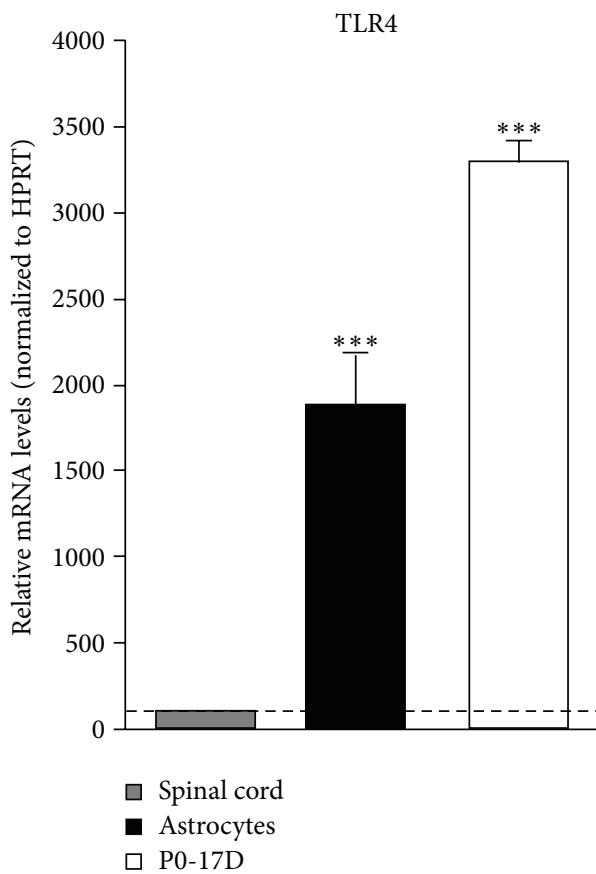

FIgURE 1: Comparative analysis of TLR4 expression in mouse spinal cord, P0-17D cell line, and primary spinal cord astrocytes. Expression of TLR4 was determined in mouse spinal cord, P017D cells, or primary astrocytes. Total RNA was extracted, reversetranscribed, and analyzed by RT-qPCR ( $n=3$ in duplicate). Values (mean \pm s.e.m.) were normalized relative to HPRT and expressed as percentage of TLR 4 mRNA levels in mouse spinal cord $\left({ }^{* * *} p<\right.$ 0.0001 versus mouse spinal cord; one-way ANOVA followed by Bonferroni post hoc test).

Having established the most effective concentration of Withaferin A to inhibit NF- $\kappa$ B, we next switched to primary spinal cord astroglial cells. Similar to P0-17D cells, we confirmed higher levels of TLR4 mRNA in primary astrocytes when compared to those in mouse spinal cord by RT-qPCR (Figure 1). The effect of Withaferin A towards LPS-induced NF- $\kappa \mathrm{B}$ activity was then explored in cultured spinal cord astrocytes transiently transfected with the NF- $\kappa \mathrm{B}-\mathrm{RE}-\mathrm{Luc}$ transgene. Cells were treated with Withaferin $\mathrm{A}(0.5 \mu \mathrm{M})$ for 1 hour, followed by stimulation with LPS $(1 \mu \mathrm{g} / \mathrm{mL}, 6$ hours $)$. Using the luciferase reporter assay and immunocytochemical analysis, we could confirm the ability of this natural compound to inhibit the transcriptional activity of NF- $\kappa \mathrm{B}$ (Figure 3(a)) as well as the nuclear translocation of its p65 subunit (Figure 3(c)) triggered by LPS treatment. Also in this 
NF- $\kappa$ B

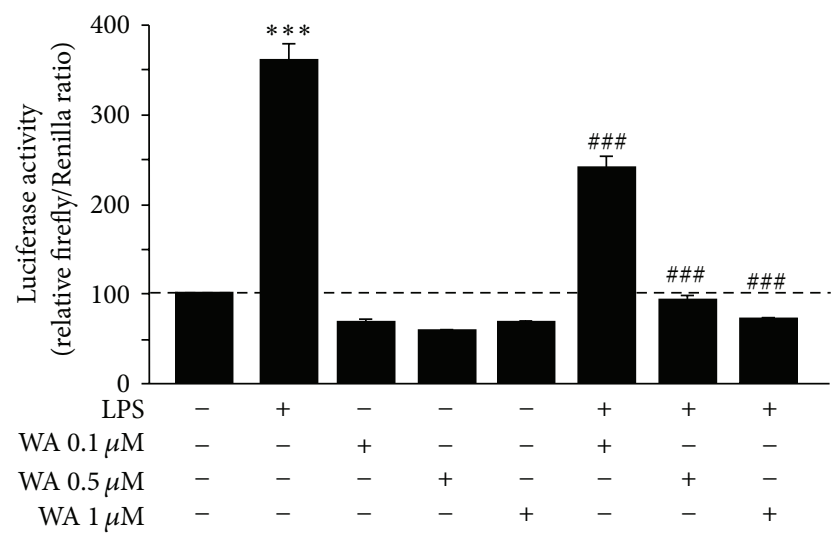

(a)

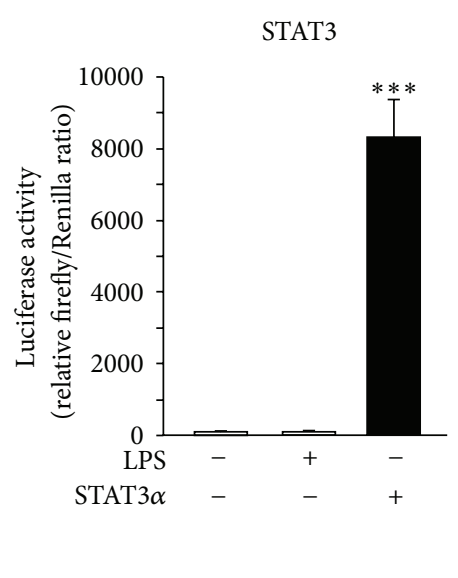

(b)

FIGURE 2: Impact of Withaferin A on LPS-induced NF- $\kappa$ B activity in immortalized P0-17D cortical astrocytes. (a) P0-17D astrocytic cells were transiently co-transfected with the NF- $\kappa \mathrm{B}-\mathrm{RE}-\mathrm{Luc}$ transgene and the Renilla luciferase encoding plasmid, used as transfection control. Twenty-four hours after the transfection, cells were pre-treated for 1 hour with increasing concentrations of Withaferin A (WA) and then incubated in the presence or in the absence of $1 \mu \mathrm{g} / \mathrm{mL}$ LPS for additional 6 hours ( $n=3$ experiments in triplicate). Cell lysates were assayed for luciferase enzymatic activities, and firefly luciferase levels were normalized to Renilla luciferase values. Data (mean \pm s.e.m.) are expressed as percentage of luciferase activity in control conditions, that is, the corresponding culture type challenged with saline $\left({ }^{* * *} p<0.0001\right.$ versus control, ${ }^{\# \# \# ~} p<0.0001$ versus LPS; one-way ANOVA followed by Bonferroni post hoc test). (b) P0-17D astrocytes were transiently co-transfected with STAT3-driven firefly luciferase and Renilla luciferase reporter vectors in the absence or in the presence of a plasmid encoding the constitutively active STAT $3 \alpha$ protein. Cells were subsequently incubated with or without $1 \mu \mathrm{g} / \mathrm{mL}$ LPS for 6 hours $(n=3$ experiments in triplicate) and cell lysates were assayed for luciferase enzymatic activities, as above. Data (mean \pm s.e.m.) are expressed as percentage of luciferase activity in control conditions, that is, the corresponding culture type challenged with saline $\left({ }^{* * *} p<0.0001\right.$ versus control; one-way ANOVA followed by Bonferroni post hoc test).

case, Withaferin A alone did not interfere with the basal activity of NF- $\kappa$ B (Figure 3(a)). Besides, we could exclude that the effect of Withaferin A was linked to the activity of STAT3, because LPS could not activate this factor in spinal cord astrocytes (Figure 3(b)).

$\mathrm{NF}-\kappa \mathrm{B}$ is notoriously a versatile transcription factor that controls the expression of several pro-inflammatory and stress response mediators [15]. These include, for example, the cytokine Tumor Necrosis Factor $\alpha$ (TNF $\alpha)$, the inducible isoform of the cyclooxygenase enzyme (COX-2), which is deputed to the production of eicosanoids (e.g., prostaglandins), and the inducible isoform of nitric oxide synthase (iNOS), which mediates the production of nitric oxide (NO) [33-36]. Remarkably, these proteins are widely considered key effectors of the neuroinflammatory reaction that characterizes several neurological disorders [37, 38]. In addition, considerable evidence indicates that they can contribute to excitotoxic neuronal cell death in vitro [3942]. Thus, the identification of pharmacological compounds that inhibit their production is highly desirable. On these bases, we next decided to investigate the impact of LPS and Withaferin A towards the expression of these mediators in primary spinal cord astrocytes by RT-qPCR. Astroglial cells in culture were pre-treated with SC-514, a specific NF- $\kappa \mathrm{B}$ inhibitor, or the most effective dose of Withaferin $\mathrm{A}(0.5 \mu \mathrm{M})$ for 1 hour and, then, exposed to LPS ( $1 \mu \mathrm{g} / \mathrm{mL} ; 6$ hours). Gene expression analysis revealed that the levels of TNF $\alpha, \mathrm{COX}$ 2 , and iNOS mRNAs significantly increased after stimulation with the endotoxin (Figure 4), thus confirming the capacity of our cell model system to generate immune responses in vitro. In addition, we found that both SC-514 and Withaferin A significantly reduced the increment in TNF $\alpha, \mathrm{COX}-2$, and iNOS expression levels triggered by LPS stimulation. Given the prophylactic anti-inflammatory efficacy of Withaferin A in vitro, we next decided to explore the therapeutic potential of this natural compound. To this end, spinal cord astrocytes were exposed to LPS $(1 \mu \mathrm{g} / \mathrm{mL})$ for six hours, as above. Two hours after the start of the incubation, cells were supplemented with Withaferin A $(0.5 \mu \mathrm{M})$ and incubated for additional 4 hours. RT-qPCR analysis confirmed that the post-treatment with Withaferin $\mathrm{A}$ is able to potently reduce the levels of mRNAs coding for TNF $\alpha, \mathrm{COX}-2$, and iNOS induced by LPS stimulation (Figure 5). No significant impact of Withaferin A itself on the basal expression of the three genes was detected after both the pre-treatment and the posttreatment when compared to control conditions (Figures 4 and 5).

Altogether, these data confirm that the expression of the three mediators induced by LPS is dependent on the activity of NF- $\kappa$ B in spinal cord astrocytes. Furthermore, they highlight a role for Withaferin $\mathrm{A}$ in reducing the $\mathrm{NF}-\kappa \mathrm{B}-$ dependent production of these neurodamaging factors (Figures 4 and 5). Although Withaferin A was previously shown to inhibit STAT3 in other cell types [22-24], we demonstrate that its anti-inflammatory effect cannot be ascribed to this mechanism, as we reveal that STAT3 activity is insensitive to the action of LPS in both P0-17D and primary spinal cord astrocytes (Figures 2(b) and 3(b)). 


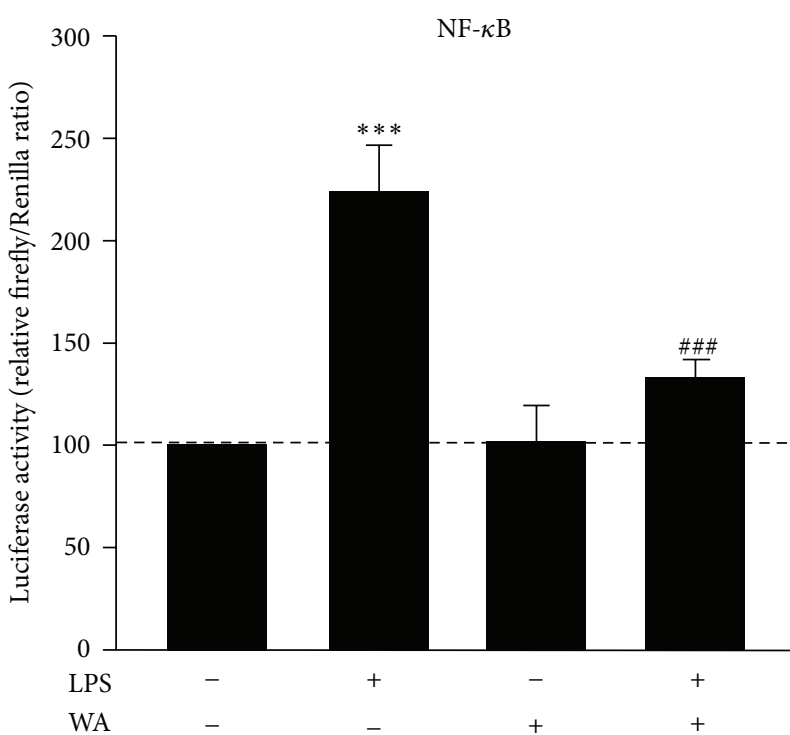

(a)

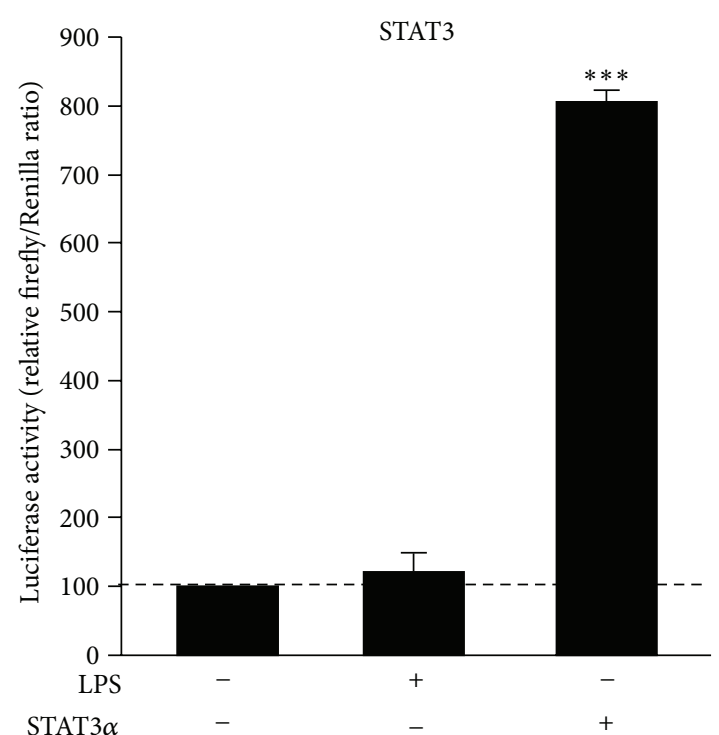

(b)
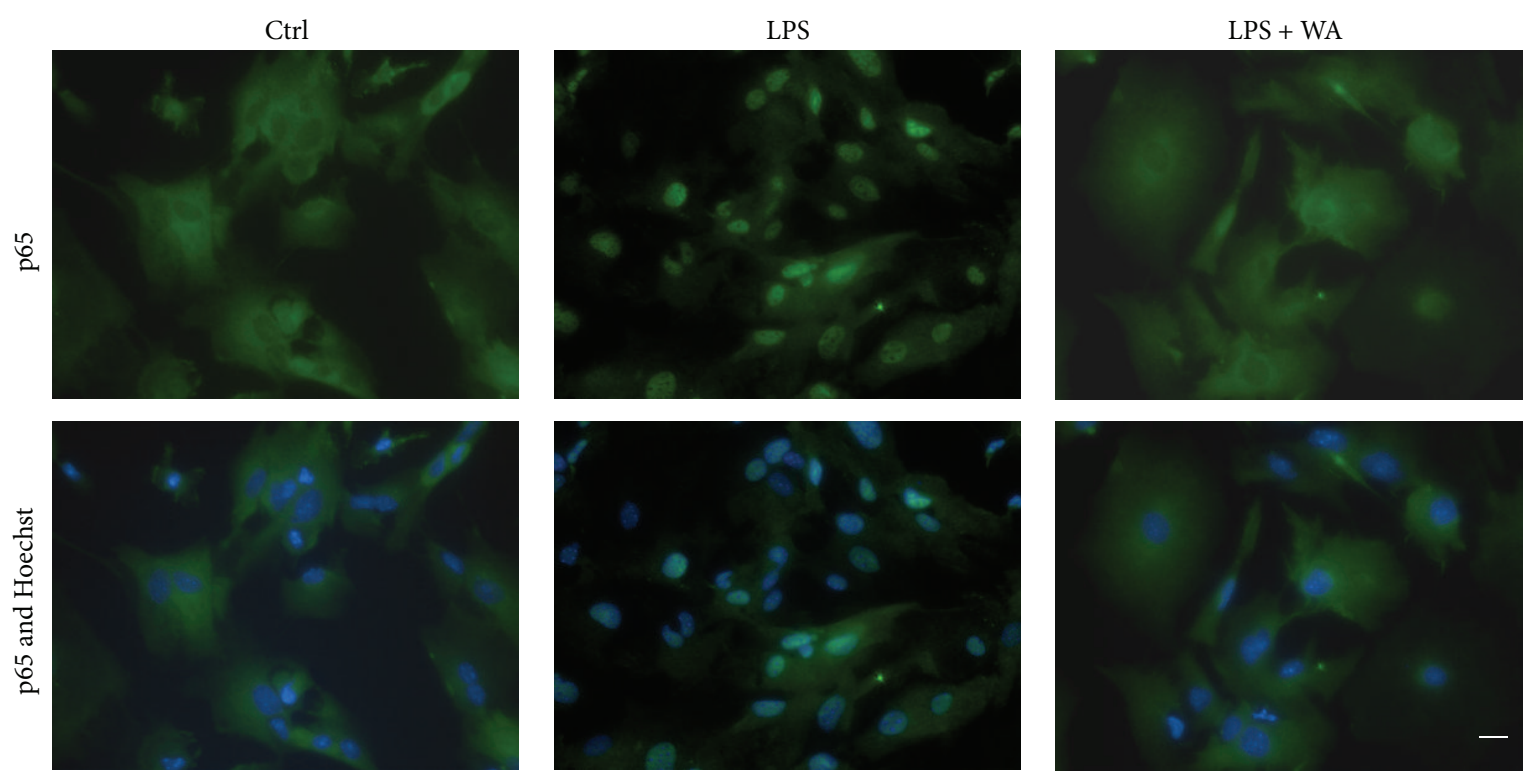

(c)

FIGURE 3: Effects of Withaferin A on LPS-induced NF- $\kappa$ B activity in primary spinal cord astrocytes. (a) Primary astrocytes from the spinal cord of newborn mice were transiently co-transfected with NF- $\kappa$ B-driven firefly and Renilla luciferase reporter vectors. Twenty-four hours after the transfection, cells were preincubated in the presence or in the absence of $0.5 \mu \mathrm{M}$ Withaferin A (WA) for 1 hour and then treated with or without $1 \mu \mathrm{g} / \mathrm{mL}$ LPS for additional 6 hours ( $n=4$ experiments in quintuplicate). Cell lysates were assayed for luciferase enzymatic activities, and firefly luciferase levels were normalized to Renilla luciferase values. Data (mean \pm s.e.m.) are expressed as percentage of luciferase enzymatic activity in control conditions $\left({ }^{* * *} p<0.0001\right.$ versus control, ${ }^{\# \#} p<0.0001$ versus LPS; one-way ANOVA followed by Bonferroni post hoc test). (b) Primary astrocytes were transiently co-transfected with the STAT3-driven firefly and Renilla luciferase reporter vectors in the presence or in the absence of a plasmid encoding the STAT3 $\alpha$ activator protein. Cells were subsequently incubated with or without $1 \mu \mathrm{g} / \mathrm{mL}$ LPS for 6 hours ( $n=3$ experiments in triplicate). Cell lysates were assayed for luciferase enzymatic activities, as above. Data (mean \pm s.e.m.) are expressed as percentage of luciferase activity in control conditions $\left({ }^{* * *} p<0.0001\right.$ versus control; one-way ANOVA followed by Bonferroni post hoc test). (c) Astrocytic cultures were pre-treated in the absence or in the presence of $0.5 \mu \mathrm{M}$ WA for 1 hour followed by incubation with $1 \mu \mathrm{g} / \mathrm{mL} \mathrm{LPS}$ for 6 hours. Images are representative of cells immunolabelled for the p65 subunit of NF- $\kappa \mathrm{B}$ (green) and counterstained with Hoechst 33342 (blue) to visualise the nuclei. Scale bar, $20 \mu \mathrm{m}$. Note that the treatment with LPS induces the nuclear translocation of p65 while this effect is strongly abrogated in the presence of Withaferin A. 

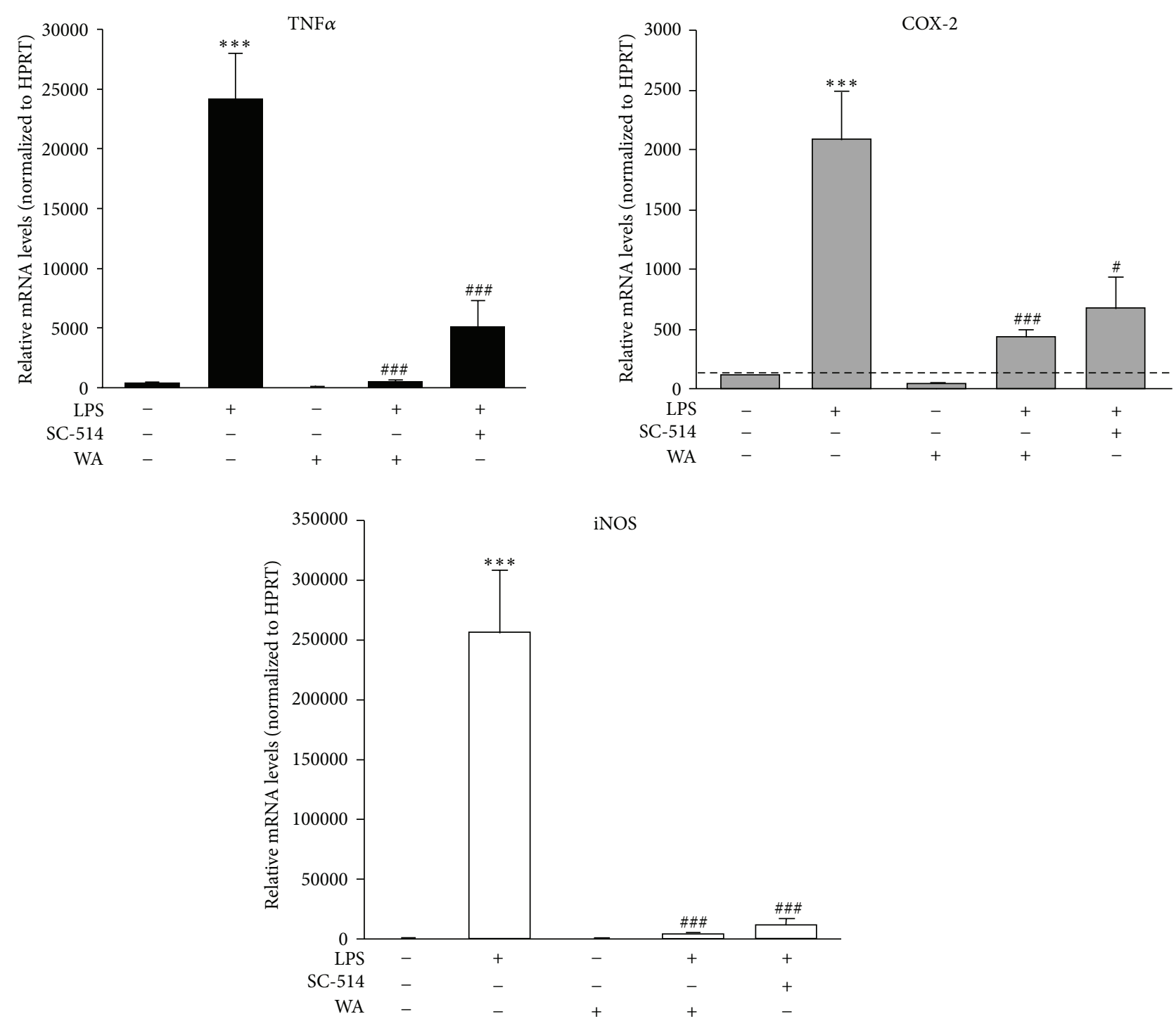

FIgURE 4: Pre-treatment with Withaferin A reduces the expression of TNF $\alpha$, COX-2, and iNOS triggered by LPS in primary spinal cord astrocytes. Primary astrocytes were pre-treated with $10 \mu \mathrm{M}$ SC-514, a selective NF- $\kappa$ B inhibitor, or $0.5 \mu \mathrm{M}$ Withaferin A (WA) for 1 hour and then incubated in the presence or in the absence of LPS $(1 \mu \mathrm{g} / \mathrm{mL})$ for 6 hours. Total RNA was extracted and reverse-transcribed. TNF $\alpha$, COX-2, and iNOS mRNA levels were analyzed by RT-qPCR ( $n=3$ experiments in triplicate). Values (mean \pm s.e.m.) were normalized relative to HPRT and expressed as percentage of TNF $\alpha$, COX-2, or iNOS expression levels in control conditions $\left({ }^{* * *} p<0.0001\right.$ versus control, ${ }^{\# \# \#} p<0.0001$ and ${ }^{\#} p<0.01$ versus LPS; one-way ANOVA followed by Bonferroni post hoc test).

\section{Conclusions}

The present work shows that activation of TLR4 in spinal cord astrocytes triggers a signaling cascade leading to NF$\kappa \mathrm{B}$ activation. The latter, in turn, promotes the expression of pro-inflammatory and stress response mediators, exemplified by $\mathrm{TNF} \alpha, \mathrm{COX}-2$, and iNOS. These events may be relevant in the context of CNS disorders as TNF $\alpha$ itself and COX2/iNOS metabolites, particularly prostaglandins and NO, can induce excitotoxic neuronal cell death in experimental models of neuroinflammation in vitro $[15,39,40]$. Furthermore, inhibition of astroglial NF- $\kappa \mathrm{B}$ in vivo by genetic approaches was reported to reduce the synthesis of inflammatory mediators in various neuropathological conditions and to prevent neurotoxic events, thus pointing at the NF- $\kappa \mathrm{B}$ pathway as a possible therapeutic target in various neurological diseases [43-46]. Our findings also demonstrate the effectiveness of Withaferin $\mathrm{A}$ in inhibiting both the transcriptional activity of NF- $\kappa \mathrm{B}$ and the consequent production of the proinflammatory and stress response mediators $\mathrm{TNF} \alpha, \mathrm{COX}-$ 2 , and iNOS in the astrocytes. Therefore, our data suggest that Withaferin A has a high therapeutic potential for the treatment of neuroinflammatory conditions characterized by a significant astrocytic contribution, and its application 

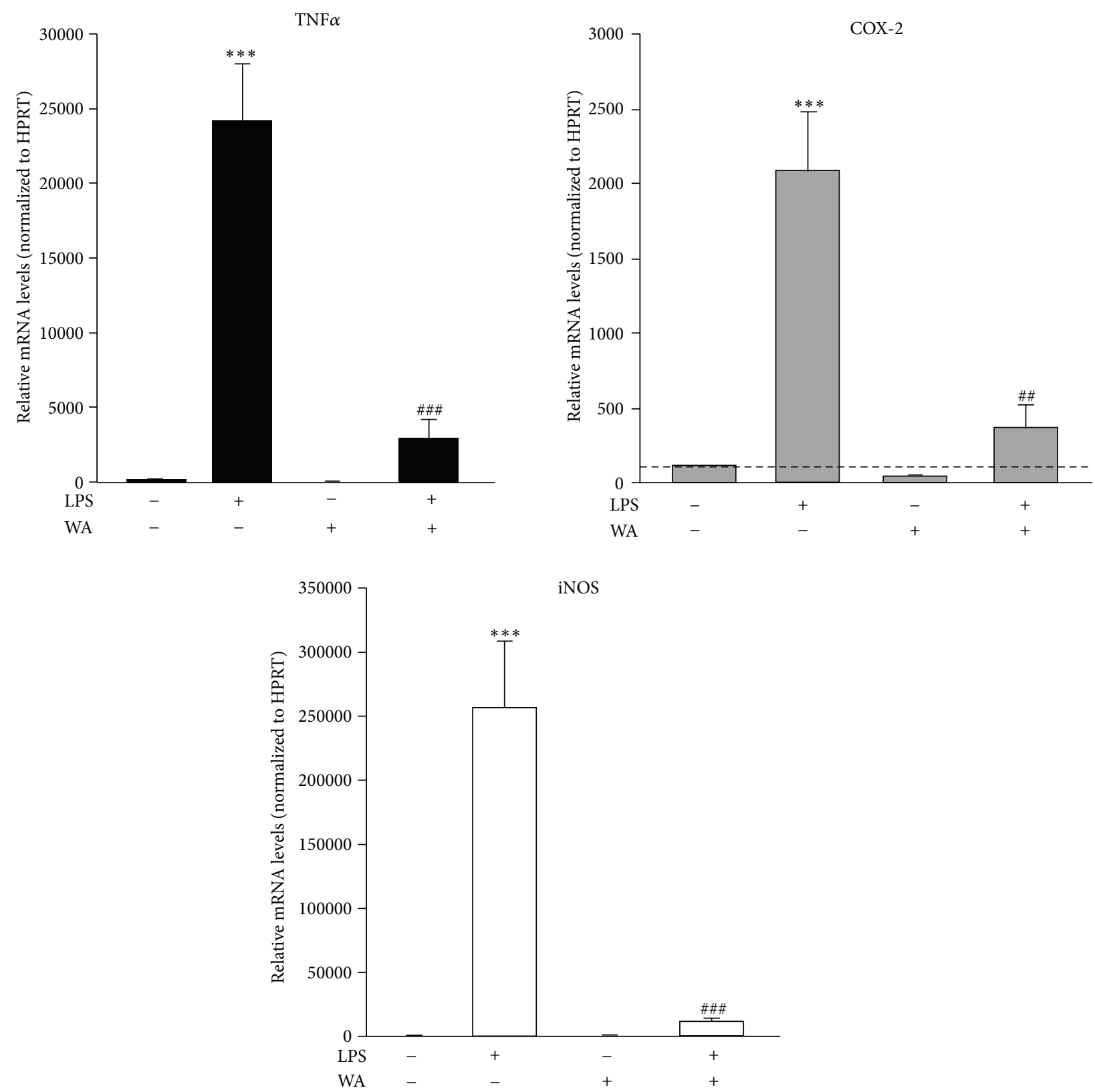

FIGURE 5: Effects of the post-treatment with Withaferin A on LPS-induced pro-inflammatory and stress response gene expression in primary spinal cord astrocytes. Primary astrocytes were treated with or without LPS $(1 \mu \mathrm{g} / \mathrm{mL})$ for 2 hours and then incubated in the absence or in the presence of $0.5 \mu \mathrm{M}$ Withaferin A (WA) plus LPS $(1 \mu \mathrm{g} / \mathrm{mL}$ ) for additional 4 hours. Total RNA was extracted and reverse-transcribed. $\mathrm{TNF} \alpha$, COX-2, and iNOS mRNA levels were analyzed by RT-qPCR $(n=2$ experiments in triplicate). Values (mean \pm s.e.m.) were normalized relative to HPRT and expressed as percentage of TNF $\alpha$, COX-2, or iNOS expression levels in control conditions $\left({ }^{* * *} p<0.0001\right.$ versus control, ${ }_{\# \# \#} p<0.0001$ and ${ }^{\# \#} p<0.001$ versus LPS; one-way ANOVA followed by Bonferroni post hoc test).

in models of neurodegenerative disorders deserves to be investigated.

\section{Conflict of Interests}

The authors declare that there is no conflict of interests regarding the publication of this paper.

\section{Acknowledgments}

The authors are grateful to Professor James W. Jacobberger (Case Western Reserve University, Cleveland, Ohio, USA) for providing the P0-17D astrocytic cell line; to Professor James Turkson (University of Hawaii, Honolulu, Hawaii, USA) and Dr. Cristina Albanesi (IDI-IRCCS, Rome, Italy) 
for providing the pLucTKS3 construct; to Dr. Ivan PlazaMenacho for providing the pSG5-STAT3 $\alpha$ plasmid (London Research Institute, London, UK); to Chiara Bergamaschi for contributions in the early phases of this study; and to Graziana Platania for experimental support. This work was supported by Regione Lombardia (Agreement no. 14501A) and Ministero della Salute (Ricerca Finalizzata 2009 RF2009-1506142) to Daniela Rossi.

\section{References}

[1] C. Farina, F. Aloisi, and E. Meinl, "Astrocytes are active players in cerebral innate immunity," Trends in Immunology, vol. 28, no. 3, pp. 138-145, 2007.

[2] R. M. Ransohoff and M. A. Brown, "Innate immunity in the central nervous system," The Journal of Clinical Investigation, vol. 122, no. 4, pp. 1164-1171, 2012.

[3] D. Rossi, "Astrocyte physiopathology: at the crossroads of intercellular networking, inflammation and cell death," Progress in Neurobiology, vol. 130, pp. 86-120, 2015.

[4] L. Brambilla, F. Martorana, and D. Rossi, "Astrocyte signaling and neurodegeneration: new insights into CNS disorders," Prion, vol. 7, no. 1, pp. 28-36, 2013.

[5] Q. Zhang, R. Kang, H. J. Zeh III, M. T. Lotze, and D. Tang, "DAMPs and autophagy: cellular adaptation to injury and unscheduled cell death," Autophagy, vol. 9, no. 4, pp. 451-458, 2013.

[6] C. K. Glass, K. Saijo, B. Winner, M. C. Marchetto, and F. H. Gage, "Mechanisms underlying inflammation in neurodegeneration," Cell, vol. 140, no. 6, pp. 918-934, 2010.

[7] M. Bsibsi, R. Ravid, D. Gveric, and J. M. van Noort, "Broad expression of Toll-like receptors in the human central nervous system," The Journal of Neuropathology \& Experimental Neurology, vol. 61, no. 11, pp. 1013-1021, 2002.

[8] C. C. Bowman, A. Rasley, S. L. Tranguch, and I. Marriott, "Cultured astrocytes express toll-like receptors for bacterial products," Glia, vol. 43, no. 3, pp. 281-291, 2003.

[9] P. A. Carpentier, W. S. Begolka, J. K. Olson, A. Elhofy, W. J. Karpus, and S. D. Miller, "Differential activation of astrocytes by innate and adaptive immune stimuli," Glia, vol. 49, no. 3, pp. 360-374, 2005.

[10] M. Bsibsi, C. Persoon-Deen, R. W. H. Verwer, S. Meeuwsen, R. Ravid, and J. M. Van Noort, "Toll-like receptor 3 on adult human astrocytes triggers production of neuroprotective mediators," Glia, vol. 53, no. 7, pp. 688-695, 2006.

[11] K. Hoshino, O. Takeuchi, T. Kawai et al., "Cutting edge: tolllike receptor 4 (TLR4)-deficient mice are hyporesponsive to lipopolysaccharide: evidence for TLR4 as the Lps gene product," Journal of Immunology, vol. 162, no. 7, pp. 3749-3752, 1999.

[12] A. Poltorak, X. He, I. Smirnova et al., "Defective LPS signaling in $\mathrm{C} 3 \mathrm{H} / \mathrm{HeJ}$ and $\mathrm{C} 57 \mathrm{BL} / 10 \mathrm{ScCr}$ mice: mutations in Tlr4 gene," Science, vol. 282, no. 5396, pp. 2085-2088, 1998.

[13] S. T. Qureshi, L. Larivière, G. Leveque et al., "Endotoxin-tolerant mice have mutations in toll-like receptor 4 (Tlr4)," The Journal of Experimental Medicine, vol. 189, no. 4, pp. 615-625, 1999.

[14] R. Gorina, M. Font-Nieves, L. Márquez-Kisinousky, T. Santalucia, and A. M. Planas, "Astrocyte TLR4 activation induces a proinflammatory environment through the interplay between MyD88-dependent NFkappaB signaling, MAPK, and Jak1/Stat1 pathways," Glia, vol. 59, no. 2, pp. 242-255, 2011.
[15] H. L. Pahl, "Activators and target genes of Rel/NF- $\kappa$ B transcription factors," Oncogene, vol. 18, no. 49, pp. 6853-6866, 1999.

[16] D. Singh, A. Aggarwal, R. Maurya, and S. Naik, "Withania somnifera inhibits NF-kappaB and AP-1 transcription factors in human peripheral blood and synovial fluid mononuclear cells," Phytotherapy Research, vol. 21, no. 10, pp. 905-913, 2007.

[17] M. Kaileh, W. Vanden Berghe, A. Heyerick et al., "Withaferin A strongly elicits $\mathrm{I} \kappa \mathrm{B}$ kinase $\beta$ hyperphosphorylation concomitant with potent inhibition of its kinase activity," Journal of Biological Chemistry, vol. 282, no. 7, pp. 4253-4264, 2007.

[18] A. Grover, A. Shandilya, A. Punetha, V. S. Bisaria, and D. Sundar, "Inhibition of the NEMO/IKK $\beta$ association complex formation, a novel mechanism associated with the NF-kappaB activation suppression by Withania somnifera's key metabolite withaferin A," BMC Genomics, vol. 11, no. 4, article S25, 2010.

[19] V. Khedgikar, P. Kushwaha, J. Gautam et al., "Withaferin A: a proteasomal inhibitor promotes healing after injury and exerts anabolic effect on osteoporotic bone," Cell Death and Disease, vol. 4, no. 8, article e778, 2013.

[20] R. Maitra, M. A. Porter, S. Huang, and B. P. Gilmour, "Inhibition of NFkappaB by the natural product Withaferin A in cellular models of Cystic Fibrosis inflammation," Journal of Inflammation, vol. 6, article 15, 2009.

[21] B. C. McFarland, S. W. Hong, R. Rajbhandari et al., "NF- $\kappa$ Binduced IL-6 ensures STAT3 activation and tumor aggressiveness in glioblastoma," PLoS ONE, vol. 8, no. 11, Article ID e78728, 2013.

[22] J. Lee, E.-R. Hahm, and S. V. Singh, "Withaferin A inhibits activation of signal transducer and activator of transcription 3 in human breast cancer cells," Carcinogenesis, vol. 31, no. 11, pp. 1991-1998, 2010.

[23] K.-J. Min, K. Choi, and T. K. Kwon, "Withaferin A downregulates lipopolysaccharide-induced cyclooxygenase-2 expression and PGE2 production through the inhibition of STAT1/3 activation in microglial cells," International Immunopharmacology, vol. 11, no. 8, pp. 1137-1142, 2011.

[24] H. J. Um, K.-J. Min, D. E. Kim, and T. K. Kwon, "Withaferin A inhibits JAK/STAT3 signaling and induces apoptosis of human renal carcinoma Caki cells," Biochemical and Biophysical Research Communications, vol. 427, no. 1, pp. 24-29, 2012.

[25] P. S. Frisa, M. N. Goodman, G. M. Smith, J. Silver, and J. W. Jacobberger, "Immortalization of immature and mature mouse astrocytes with SV40 T antigen," Journal of Neuroscience Research, vol. 39, no. 1, pp. 47-56, 1994.

[26] F. Martorana, L. Brambilla, C. F. Valori et al., "The BH4 domain of $\mathrm{Bcl}-\mathrm{X} \mathrm{L}$ rescues astrocyte degeneration in amyotrophic lateral sclerosis by modulating intracellular calcium signals," Human Molecular Genetics, vol. 21, no. 4, pp. 826-840, 2012.

[27] D. Rossi, L. Brambilla, C. F. Valori et al., "Focal degeneration of astrocytes in amyotrophic lateral sclerosis," Cell Death \& Differentiation, vol. 15, no. 11, pp. 1691-1700, 2008.

[28] L. Gautron, P. Lafon, M. Chaigniau, G. Tramu, and S. Layé, "Spatiotemporal analysis of signal transducer and activator of transcription 3 activation in rat brain astrocytes and pituitary following peripheral immune challenge," Neuroscience, vol. 112, no. 3, pp. 717-729, 2002.

[29] Y. Nakano, E. Furube, S. Morita, A. Wanaka, T. Nakashima, and S. Miyata, "Astrocytic TLR4 expression and LPS-induced nuclear translocation of STAT3 in the sensory circumventricular organs of adult mouse brain," Journal of Neuroimmunology, vol. 278, pp. 144-158, 2015. 
[30] E. Beurel and R. S. Jope, "Lipopolysaccharide-induced interleukin- 6 production is controlled by glycogen synthase kinase- 3 and STAT3 in the brain," Journal of Neuroinflammation, vol. 6, article 9, 2009.

[31] Y.-J. Lee, D.-Y. Choi, I. S. Choi et al., "Inhibitory effect of a tyrosine-fructose Maillard reaction product, 2,4-bis(p-hydroxyphenyl)-2-butenal on amyloid-beta generation and inflammatory reactions via inhibition of NF-kappaB and STAT3 activation in cultured astrocytes and microglial BV-2 cells," Journal of Neuroinflammation, vol. 8, article 132, 2011.

[32] X. Liu, Y. Tian, N. Lu, T. Gin, C. H. K. Cheng, and M. T. V. Chan, "Stat3 inhibition attenuates mechanical allodynia through transcriptional regulation of chemokine expression in spinal astrocytes," PLoS ONE, vol. 8, no. 10, Article ID e75804, 2013.

[33] D. V. Kuprash, I. A. Udalova, R. L. Turetskaya, D. Kwiatkowski, N. R. Rice, and S. A. Nedospasov, "Similarities and differences between human and murine TNF promoters in their response to lipopolysaccharide," Journal of Immunology, vol. 162, no. 7, pp. 4045-4052, 1999.

[34] Y. Kim and S. M. Fischer, "Transcriptional regulation of cyclooxygenase- 2 in mouse skin carcinoma cells. Regulatory role of CCAAT/enhancer-binding proteins in the differential expression of cyclooxygenase-2 in normal and neoplastic tissues," Journal of Biological Chemistry, vol. 273, no. 42, pp. 27686-27694, 1998.

[35] S. T. Reddy, D. J. Wadleigh, and H. R. Herschman, “Transcriptional regulation of the cyclooxygenase- 2 gene in activated mast cells," The Journal of Biological Chemistry, vol. 275, no. 5, pp. 3107-3113, 2000.

[36] Q.-W.Xie, R. Whisnant, and C. Nathan, "Promoter of the mouse gene encoding calcium-independent nitric oxide synthase confers inducibility by interferon $\gamma$ and bacterial lipopolysaccharide," The Journal of Experimental Medicine, vol. 177, no. 6, pp. 1779-1784, 1993.

[37] M. T. Heneka, D. T. Golenbock, and E. Latz, "Innate immunity in Alzheimer's disease," Nature Immunology, vol. 16, no. 3, pp. 229-236, 2015.

[38] T. Philips and W. Robberecht, "Neuroinflammation in amyotrophic lateral sclerosis: role of glial activation in motor neuron disease," The Lancet Neurology, vol. 10, no. 3, pp. 253-263, 2011.

[39] P. Bezzi, G. Carmignoto, L. Pasti et al., "Prostaglandins stimulate calcium-dependent glutamate release in astrocytes," Nature, vol. 391, no. 6664, pp. 281-285, 1998.

[40] P. Bezzi, M. Domercq, L. Brambilla et al., "CXCR4-activated astrocyte glutamate release via TNFalpha: amplification by microglia triggers neurotoxicity," Nature Neuroscience, vol. 4, no. 7, pp. 702-710, 2001.

[41] V. L. Dawson, T. M. Dawson, E. D. London, D. S. Bredt, and S. H. Snyder, "Nitric oxide mediates glutamate neurotoxicity in primary cortical cultures," Proceedings of the National Academy of Sciences of the United States of America, vol. 88, no. 14, pp. 6368-6371, 1991.

[42] S. R. Parathath, S. Parathath, and S. E. Tsirka, "Nitric oxide mediates neurodegeneration and breakdown of the blood-brain barrier in tPA-dependent excitotoxic injury in mice," Journal of Cell Science, vol. 119, no. 2, pp. 339-349, 2006.

[43] R. Brambilla, V. Bracchi-Ricard, W.-H. Hu et al., "Inhibition of astroglial nuclear factor $\kappa \mathrm{B}$ reduces inflammation and improves functional recovery after spinal cord injury," The Journal of Experimental Medicine, vol. 202, no. 1, pp. 145-156, 2005.
[44] R. Brambilla, T. Persaud, X. Hu et al., "Transgenic inhibition of astroglial NF- $\kappa$ B improves functional outcome in experimental autoimmune encephalomyelitis by suppressing chronic central nervous system inflammation," Journal of Immunology, vol. 182, no. 5, pp. 2628-2640, 2009.

[45] E. S. Fu, Y. P. Zhang, J. Sagen et al., "Transgenic inhibition of glial NF-kappa B reduces pain behavior and inflammation after peripheral nerve injury," Pain, vol. 148, no. 3, pp. 509-518, 2010.

[46] R. Brambilla, G. Dvoriantchikova, D. Barakat, D. Ivanov, J. R. Bethea, and V. I. Shestopalov, "Transgenic inhibition of astroglial NF- $\kappa$ B protects from optic nerve damage and retinal ganglion cell loss in experimental optic neuritis," Journal of Neuroinflammation, vol. 9, article 213, 2012. 

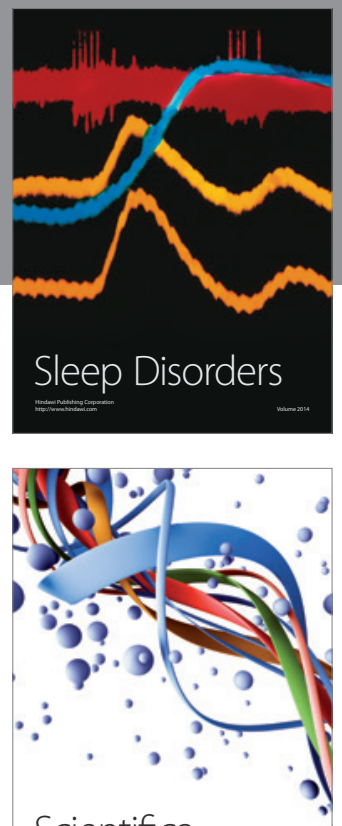

Scientifica
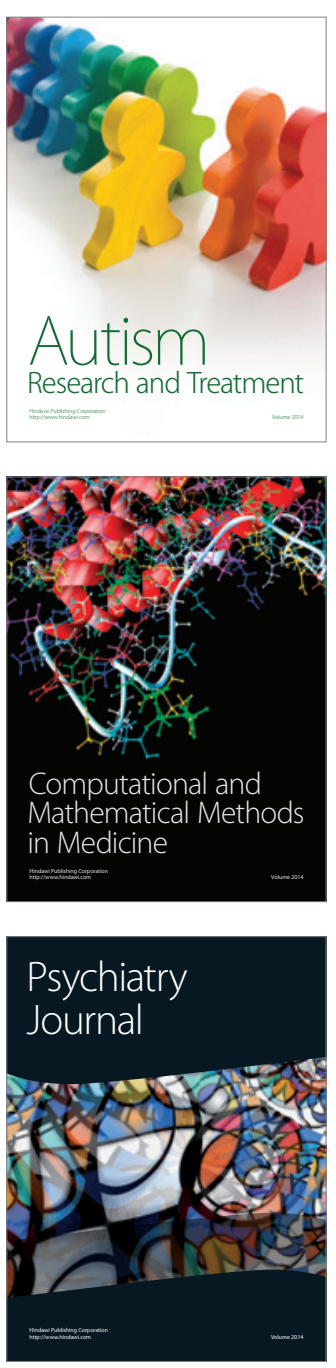
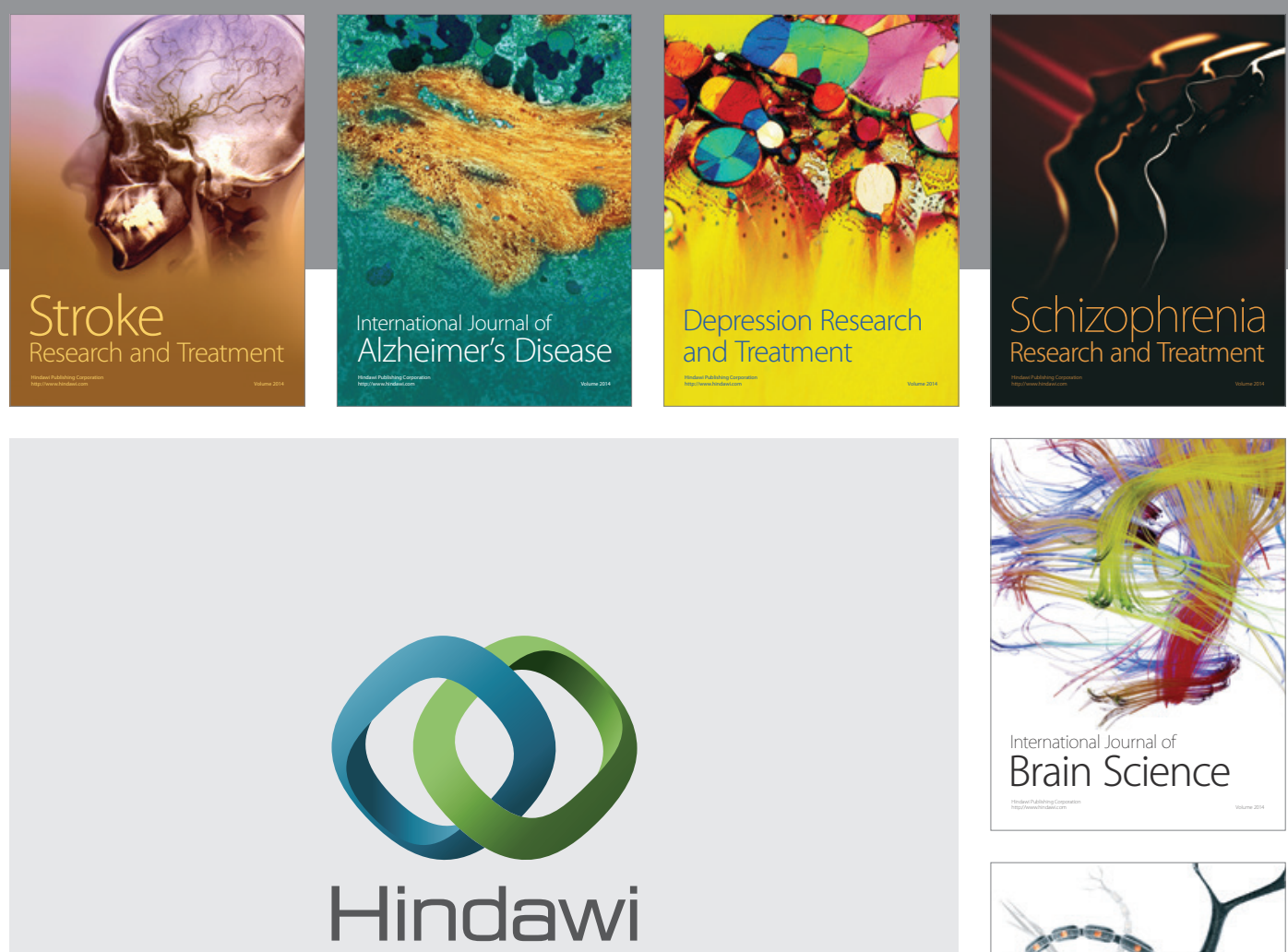

Submit your manuscripts at

http://www.hindawi.com
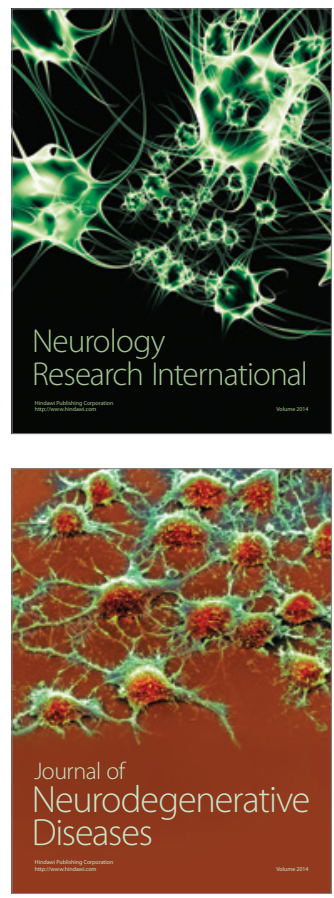

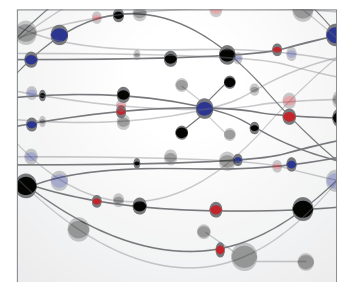

The Scientific World Journal
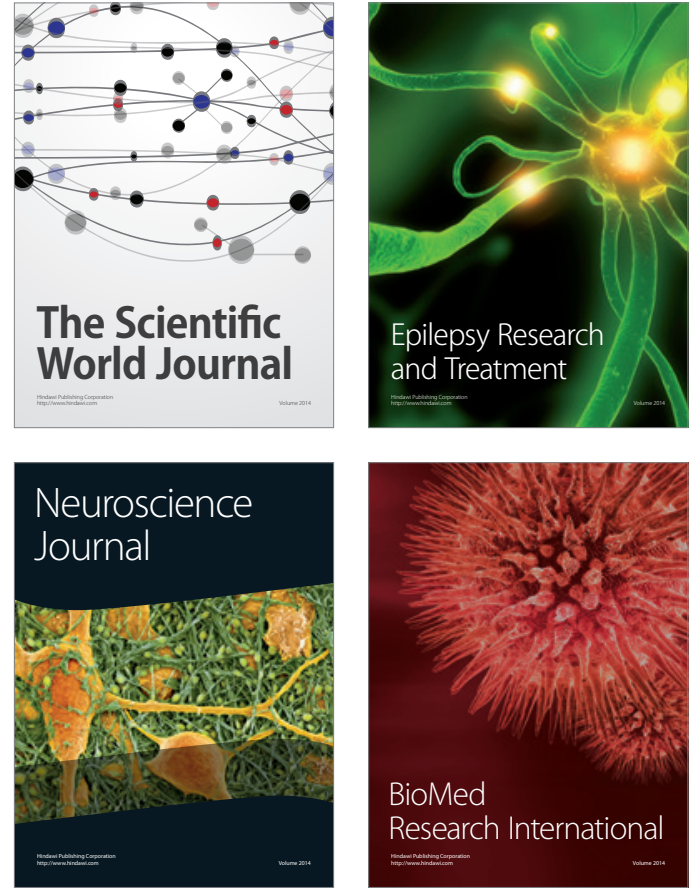

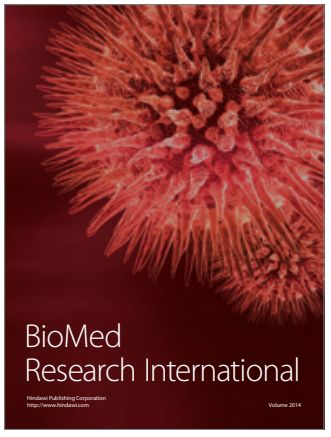

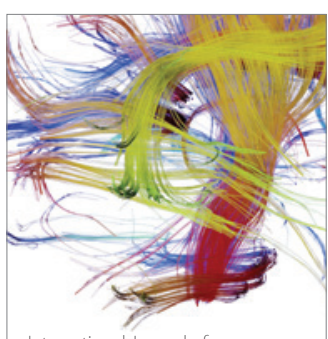

Brain Science

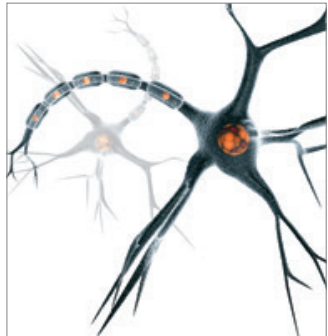

Neural Plasticity
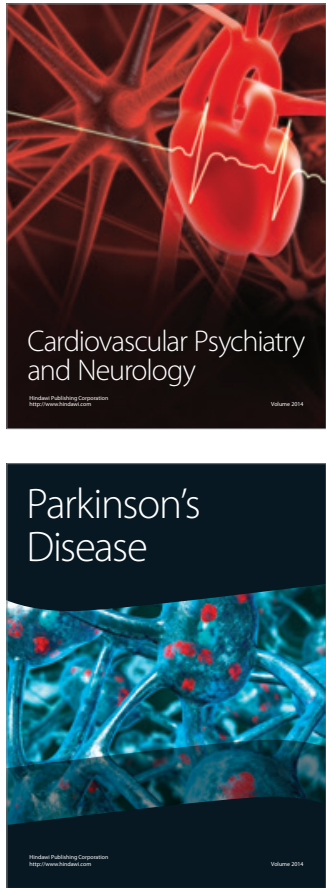